\title{
Contributions of vitamin $D$ in the management of depressive symptoms and cardiovascular risk factors: study protocol for a randomized, double-blind, placebo- controlled clinical trial
}

\author{
Catarina Magalhães Porto ${ }^{*}$, Tatiana de Paula Santana da Silva and Everton Botelho Sougey
}

\begin{abstract}
Background: Depression is a major cause of chronic disability throughout the world and an important cardiovascular risk factor, increasing the relative risk of coronary artery disease as well as rates of cardiovascular morbidity and mortality. Concomitant to the high prevalence of depression, there has been a reduction in exposure to sunlight with the increase in urbanization and the use of sun protectors, which has led to a reduction in serum levels of 25-hydroxyvitamin D. Therefore, this paper describes a protocol for a clinical trial with the aim of evaluating the effects of vitamin D supplementation on depression and cardiovascular risk factors to contribute evidence regarding the potential influence of supplementation on mood regulation.
\end{abstract}

Methods: This protocol study was guided by the Standard Protocol Items: Recommendations for Interventional Trials. A randomized, placebo-controlled, double-blind clinical trial will be conducted involving 224 adults (age range 18-60 years) with depression who are taking antidepressants and have no history of vitamin D supplementation, psychiatric comorbidities, chronic kidney disease, hypercalcemia, or neoplasm. The participants will be recruited from the psychiatric outpatient clinics of two universities in northeast Brazil. Eligible participants who provide written consent will be randomly designated to either the intervention group ( $n=112$; vitamin $D$ supplementation 50,000 IU per week for 6 months) or the control group ( $n=112$; placebo taken weekly for 6 months). Measures for monitoring depressive symptoms, clinical examinations, and laboratory tests for evaluating cardiovascular risk factors and serum vitamin D levels will be performed before and after the intervention period.

Discussion: To the best of our knowledge, this will be the first clinical trial with the aim of testing the effectiveness of vitamin D supplementation on the reduction of cardiovascular risk and as an adjuvant to depression therapy for a prolonged period (6 months). The findings will contribute to the understanding of the therapeutic effects of vitamin D supplementation in the management of depression and can help guide public policies directed toward vitamin supplementation for the reduction of cardiovascular risk.

Trial registration: Brazilian Clinical Trials Registry, RBR-6yj8sj/Universal Trial Number (UTN) U1111-1217-9237. Registered on 23 July 2018.

Keywords: Vitamin D, Depressive symptoms, Major depressive disorder, Cardiovascular disease, Clinical trial

\footnotetext{
* Correspondence: catarinamqo@gmail.com

Neuropsychiatry and Behavioral Sciences, Federal University of Pernambuco

(UFPE), Av. Prof. Moraes Rego, 1235, Cidade Universitária, Recife, PE CEP:

50670-901, Brazil
} 


\section{Background}

Depression is a major cause of disability throughout the world, with a report of more than 300 million affected individuals in 2015, which corresponds to $4.4 \%$ of the global population [1]. Depression is also considered a cardiovascular risk factor, increasing the relative risk of coronary artery disease as well as rates of cardiovascular morbidity and mortality [2-4]. As depression is a complex disorder that likely has multiple subtypes and etiologies, calcitriol (vitamin D) could play an important role in this condition [5].

Vitamin D receptors have been identified in areas of the brain involved with depression, such as the prefrontal cortex, hypothalamus, and substantia nigra. Therefore, this vitamin is considered a neurosteroid. Vitamin D has also been found to increase the expression of genes that code tyrosine hydroxylase, which is a precursor of dopamine and norepinephrine [5]. Moreover, calcitriol may provide significant protection against the effects of the reduction in neurotransmitters (dopamine and serotonin) at neurotoxic doses of methamphetamine. Calcitriol has been found to play an important role in neurons in both in vitro and in vivo studies and has the ability to regulate trophic factors and protect against various lesions [6].

Many studies have suggested that psychiatric disorders, such as schizophrenia, alcoholism, and depression, may be associated with low serum levels of 25hydroxyvitamin D, 25(OH)D [7-13]. Recent studies report that vitamin $\mathrm{D}$ deficiency may be associated with an increase in depression rates from 8 to 14\% [14-19].

In addition, a double-blind, placebo-controlled study showed that vitamin D supplementation for 8 weeks was beneficial but not significant $(p=0.06)$ in reducing symptoms of depression, demonstrating a possible tendency of this hormone action on handling of depression. Nevertheless, the authors reported an improvement in pancreatic $\beta$-cell insulinemia and homeostasis, also interfering with the total antioxidant capacity of plasma [20]. Recent meta-analyses conducted in accordance with the Preferred Reporting Items for Systematic Reviews and Meta-Analyses (PRISMA) report a statistically significant reduction in depressive symptoms and clinically significant reduction in depression following vitamin D supplementation [21, 22].

This article presents the protocol for a randomized, placebo-controlled, double-blind clinical trial with the aim of evaluating the therapeutic effects of vitamin D (50,000 IU per week for 6 months) on depression and cardiovascular risk factors in patients with depression in care at the psychiatric clinics of two university hospitals in northeast Brazil compared to a control group (placebo once per week for 6 months). The hypothesis is that vitamin D supplementation is capable of improving depressive symptoms and lowering both the risk of suicide and cardiovascular risk in patients with depression.

\section{Methods}

This protocol study was guided by the Standard Protocol Items: Recommendations for Interventional Trials (SPIRIT) statement (see Fig. 1, the flowchart of the study and Additional file 1, the SPIRIT checklist). The proposed prospective, randomized, placebo-controlled, double-blind trial has been duly registered (http://www. ensaiosclinicos.gov.br/rg/ with trial registry number RBR-6yj8sj and Universal Trial Number (UTN) U11111217-9237, July 23, 2018).

The clinical trial will be conducted in accordance with the precepts stipulated in the Declaration of Helsinki, and the project received approval from the Human Research Ethics Committee of the Federal University of Pernambuco (certificate number 36379347717.0.0000.5208 from January 11, 2018). All participants will be recruited from the psychiatric outpatient clinics of the University of Pernambuco and Federal University of Pernambuco. We will evaluate 224 male and female adults (18-60 years of age) with depression under routine care at the psychiatric clinics for a period of 6 months.

\section{Eligibility criteria}

Individuals between 18 and 60 years of age with a diagnosis of depression (score $>6$ points on the Montgomery-Asberg Depression Rating Scale [23]) will be recruited independently of sex or gender. The participants will need to have a diagnosis of depression provided by a psychiatrist and based on the ICD-10 [24], such as F33.0 (recurring mild depression), F33.1 (recurring moderate depressive disorder), F33.2 (severe recurring depressive disorder without psychotic symptoms, including recurring major depression, endogenous depression, and manic-depressive psychosis in the depressive form without psychotic symptoms), F33.3 (severe depressive disorder with psychotic symptoms, including major depression with psychotic symptoms, psychotic depression, manic-depressive psychosis in the depressive form with psychotic symptoms), F33.8 (other recurring depressive disorders), and F33.9 (recurring severe depressive disorder without psychotic symptoms, including recurrent major depression without specification or unipolar depression).

The patients may be taking an antidepressant prescribed by their psychiatrists. No restriction will be imposed regarding the type of antidepressant medication. The severity of the disease may be mild, moderate, or severe, as evaluated by the psychiatrist.

Individuals with cognitive impairment (determined using the Mini-Mental Health Examination [25]), those already supplementing with vitamin $\mathrm{D}$, those with a past 


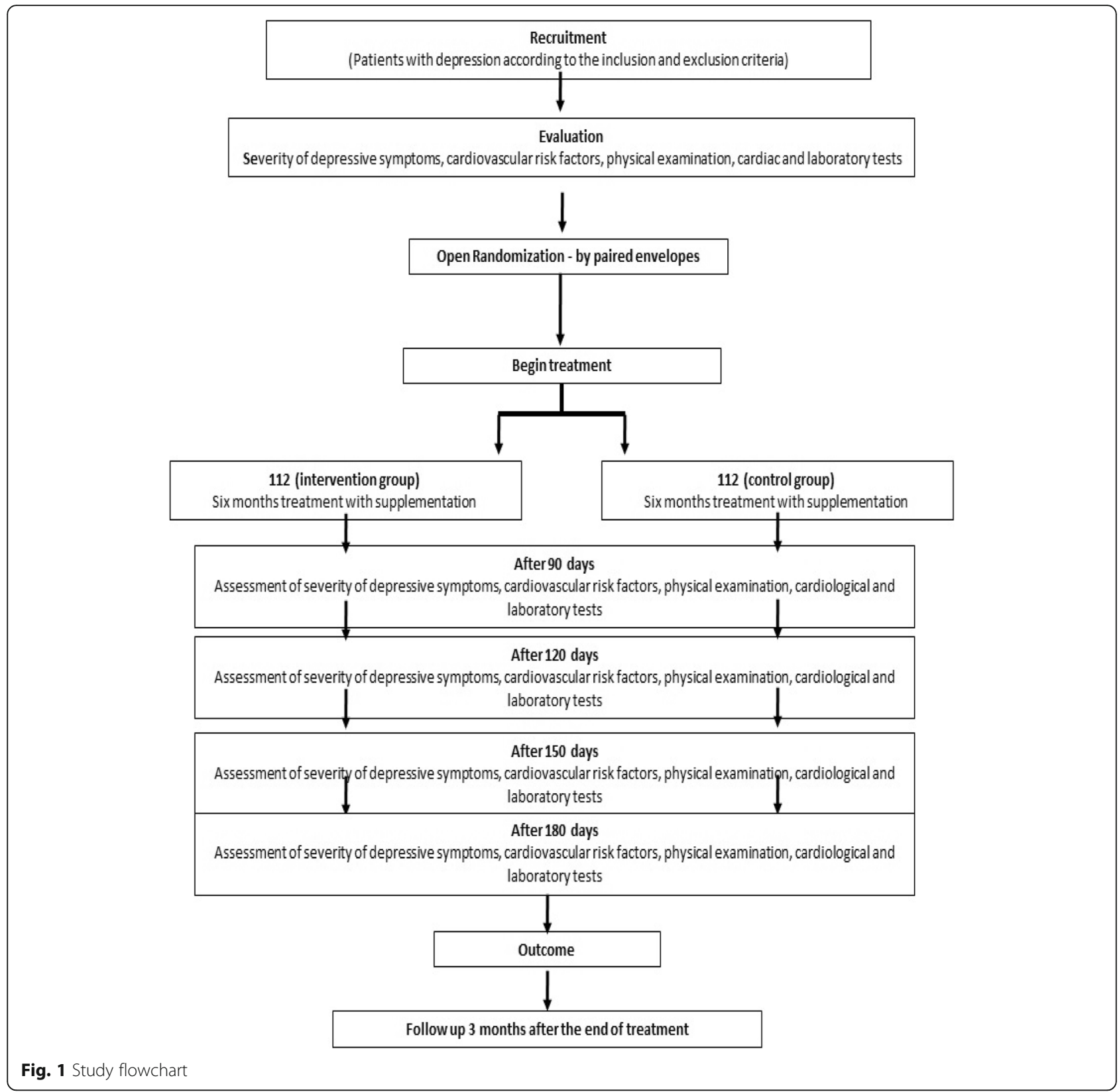

history of psychiatric comorbidities, chronic kidney disease, hypercalcemia, or neoplasms, and pregnant or nursing women will be excluded from the study.

\section{Operational procedures}

Two pharmacists will perform the randomization and will be aware of which patients are using vitamin D and which are using the placebo. The participants will be divided into two groups (A and B). The main researcher of the clinical trial will interview all participants and fill out the clinical charts. A nurse will administer the vitamin D and placebo but will not know which substance is being given to the participants. The patients and investigator will be unaware of which capsule (vitamin D or placebo) the patients are taking. This procedure is necessary to avoid observation bias during the clinical laboratory follow-up and maintain complete impartiality in the evaluation of the effects.

All instructions will be precise and written in the form of a manual to ensure the execution of all procedures determined for the clinical trial, including patient recruitment, allocation to the study groups, administration of the intervention, record systems, criteria for interrupting the intervention, etc. All activities to be performed 
during the clinical trial will be previously established in the form of a task list distributed to the investigation team.

The initial evaluation will consist of completion of the identification chart, sociodemographic inventory, standardized medical chart with patient history, physical and cardiological examinations, a questionnaire addressing the practice of activities involving exposure to sunlight, standardized statement of informed consent, a structured interview addressing depression with the use of the Montgomery-Asberg Depression Rating Scale, a scale addressing the risk of suicide, and the recording of the results of laboratory and cardiological exams. The researcher will accompany the examinations and administration of the depression and suicidal risk scales.

The laboratory tests will be conducted at the laboratory of the hospital in which the study will be developed. Venous blood $(10 \mathrm{ml})$ will be collected from a peripheral vein for the determination of fasting glucose, cholesterol (total and fractions), urea, creatinine, total proteins, albumin, homocysteine, ultrasensitive C-reactive protein, lipoprotein A, ionic calcium, and phosphorus as well as hormonal levels of total and free testosterone, estradiol, estriol, luteinizing hormone, follicle-stimulating hormone, thyroid-stimulating hormone (TSH), triiodothyronine, free tetraiodothyronine, cortisol, concentration of 25-hydroxyvitamin D and parathyroid-stimulating hormone upon admission to the study and every 90 days for a total period of 6 months. Urine will be collected every 90 days for the determination of 24-h calciuria and microalbuminuria. The following laboratory equipment will be used for the determinations: Architect C8000 for vitamin D and hormone concentrations and CMD 800 with ISE (Wiener Group) for the biochemical concentrations.

Cardiological and imaging exams (electrocardiography, Doppler echocardiography, stress test, electrocardiographic monitoring [24-h Holter], and Doppler ultrasound of the carotid and vertebral arteries) will be performed at the hospital in which the study will be developed.

When the participants return with the exam results, simple randomization (heads or tails) will be performed for the allocation to the different groups: 112 individuals in the vitamin $D_{3}$ group and 112 in the placebo group. A nurse at the clinic will administer the vitamin $D_{3}$ and placebo. The capsules will have the same size and color (compounded for the clinical trial), and the nurse will be blinded to the allocation of the patients to the different groups.

The researchers will evaluate the participants every 90 days. Each evaluation will involve the clinical and cardiological examinations with the electrocardiography and laboratory tests selected for the study. The final evaluation will occur after 6 months of intervention. The participants will be submitted to psychiatric testing (Montgomery-Asberg Depression Rating Scale [23] and Columbia-Suicide Severity Rating Scale [26]), clinical and cardiological examinations, as well as the laboratory tests, electrocardiography, Doppler echocardiography, a stress test, 24-h Holter, and Doppler ultrasound of the carotid and vertebral arteries.

As the region in which the clinical trial will be conducted is sunny throughout the entire year, with an absence of well-defined climatic seasons, seasonality will not exert an influence on exposure to sunlight or the synthesis of vitamin D [27].

\section{Calculation of sample size}

The following were considered for the determination of the sample size:

1. The comparative objective will be an improvement in the depression score among the patients taking and those not taking vitamin D. We considered the success percentages obtained in a study on vitamin D [28] in which $69.2 \%$ and $65 \%$ improvements were found in the depression scale scores, respectively.

2. We assumed $5.0 \%$ acceptable rate of error, $80.0 \%$ power, and a ratio of 1.00 between the two groups.

The necessary sample size for each group was 112 patients (total of 224 patients). This calculation was performed using the Epi Info program, version 6.04.

\section{Reduction of risk of bias}

The dosage of vitamin $\mathrm{D}$ for supplementation considered for the study $(50,000 \mathrm{IU}$ weekly) meets the preestablished criteria in previous trials that have presented positive results in the reduction of depressive symptoms [20]. The aim of considering this variable is to minimize the potential bias. Vitamin $\mathrm{D}_{3}$ is quite safe when administered with the prescribed posology. Dosages up to 10, $000 \mathrm{IU}$ per day for 5 months induced no signs of toxicity, such as hypercalcemia and hypercalciuria $[29,30]$.

\section{Collection and management of data, including meta-data}

The characteristics and procedures adopted for each test group are as follows:

Group 1 - Intervention: 112 participants will receive vitamin $\mathrm{D}_{3}$ supplementation (Addera D3, Mantecorp Laboratory) at a dosage of 50,000 IU per week for 6 months.

Group 2 - Placebo: 112 participants will receive a placebo capsule (compounded by the pharmaceutical laboratory of the Federal University of Pernambuco) once a week for 6 months. 
The groups will be submitted to five evaluations on the first day and after 6 months of intervention:

1. Questionnaire addressing sociodemographic characteristics. The questionnaire will be administered to the participants in interview form and filled out by the researcher. The following data will be recorded: identification number attributed to the participant, age, sex, schooling, marital status, ethnicity, and religion.

2. Brazilian Economic Classification Criteria (BECC) 2011 established by the Brazilian Association of Companies in Research [31]. The BECC is an economic segmentation instrument that uses a survey of residential characteristics (presence and quantity of household items of comfort and degree of schooling of the head of the family) to

differentiate the population. Points are attributed to each household characteristic, and the total is used to classify economic strata from highest to lowest class: A1, A2, B1, B2, C1, C2, D, E [31].

3. Severity of depression. This is determined through a structured clinical interview. The Montgomery-

Asberg Depression Rating Scale [23] will be administered at the beginning of the study and after 6 months of intervention.

4. Assessment of risk of suicide. The Columbia-Suicide Severity Rating Scale [26] will be administered to assess the presence and intensity of suicidal ideation and suicidal behavior.

5. Cardiovascular risk factors. These are (1) systemic arterial hypertension based on systolic and diastolic blood pressure levels [32]; (2) dyslipidemia (total cholesterol, high- and low-density lipoproteins [HDL, LDL] triglycerides) [33]; (3) coronary artery disease: coronary calcium $>100$ th or $>75$ th percentile for age or sex, cardiac stress test or myocardial scintigraphy with signs of myocardial ischemia [34]; (4) cerebrovascular accident (stroke): clinical condition or suggestive imaging exam; (5) obstructive peripheral arterial disease: anklebrachial pressure index $<0.9$; arterial stenosis detected by ultrasound of lower limbs [35]; (6) carotid atherosclerosis: stenosis/thickening of carotid artery [33]; (7) heart failure: left ventricular hypertrophy or reduction in systolic function of the left ventricle or diastolic dysfunction detected by Doppler echocardiography [36]; (8) arrhythmia: detected by resting electrocardiography, 24-h Holter or stress test; (9) diabetes mellitus: reduction in glucose tolerance and resistance to insulin (fasting glucose, A1C hemoglobin, oral glucose tolerance test, basal insulin, insulin curve, homeostatic model assessment) [37, 38]; (10) serum markers of cardiovascular risk (ultrasensitive C-reactive protein, homocysteine, lipoprotein A) [33]; (11) hyperthyroidism or hypothyroidism (ultrasensitive TSH, free T4, free T3) [39, 40]; (12) chronic kidney disease: albuminuria, measured by glomerular filtration rate and calculated based on the Chronic Kidney Disease Epidemiology Collaboration [41]; (13) hyperparathyroidism (HPT, phosphorus) [42].

\section{Data analysis}

The data will be analyzed using statistical measures: mean and standard deviation values for numerical variables and percentage frequencies for categorical variables. The inter-group comparisons will be performed using either the Student's $t$ test or Mann-Whitney test for numerical variables and Pearson's chi-square test for categorical variables. The intra-group comparisons of initial and final values will be performed using either the paired Student's $t$ test or the Wilcoxon test for paired data for numerical variables and McNemar's test for categorical variables.

The unpaired and paired $t$ tests will be used in situations for which the data exhibit normal distribution, whereas the Mann-Whitney test and Wilcoxon test for paired variables will be used in situations in which normality has been rejected. The Shapiro-Wilk test will be used to determine the normality of the data, and Levene's $F$ test will be used to determine equality of variances.

The margin of error used in the decisions of the statistical tests will be calculated as $5 \%$ and $95 \%$ confidence intervals. The data will be entered into an Excel spreadsheet, and the Statistical Package for the Social Sciences (SPSS version 23) will be used for all statistical calculations.

A statistician will perform the calculations and statistical analyses of the participants taking substances A and B. The blinding will only be lifted at the end of the statistical analysis.

\section{Ethical considerations}

This clinical trial is in accordance with Resolution $\mathrm{n}^{\circ}$ 466/2012 of the Brazilian National Board of Health, which establishes norms for research involving human subjects, and it received approval from the Federal University of Pernambuco (UFPE) ethics committee through Platform Brazil (certificate number 79347717.0.0000.5208) on January 11, 2018. Recruitment (screening of patients) was initiated on April 30, 2019. Thus, the study is currently in its initial phase (ongoing). The clinical trial (intervention) is expected to begin on October 1, 2019, as stipulated in the registry: RBR-6yj8sj/ UTN U1111-1217-9237. 


\section{Primary outcomes}

The primary outcomes of the trial are:

1. Therapeutic effects of vitamin D on depression, evaluated using the Montgomery-Asberg Depression Rating Scale

2. Therapeutic effects of vitamin D on cardiovascular risk factors, evaluated using the scoring system proposed by the American College of Cardiology (ACC) and American Heart Association (AHA) [43, 44] for the primary prevention of the risk of acute myocardial infarction, death by coronary heart disease, heart failure, and fatal and non-fatal stroke in a period of 10 years. The new scoring system provides specific estimates based on current age, sex, race, blood pressure, total cholesterol, HDL cholesterol, LDL cholesterol, diabetes, smoking status (current smoker, ex-smoker, or never smoked), in treatment for systemic arterial hypertension, in treatment with statins, and in treatment with aspirin. After including these data, the calculator estimates risk for cardiovascular disease in the next 10 years, which is categorized as low $(<5 \%)$, borderline $(5-7.4 \%)$, intermediate $(7.5-$ $19.9 \%)$, or high ( $\geq 20 \%)$.

\section{Secondary outcomes}

The secondary outcomes are:

1. Therapeutic effects of vitamin $\mathrm{D}$ for reducing the risk of suicide, evaluated using the ColumbiaSuicide Severity Rating Scale

2. Reduction of cardiovascular risk factors as described in the subsection "Collection and management of data, including meta-data."

\section{Analysis plan and measurement of results} The results to be measured and analyzed are the following:

1. Hypertension: reduction in systolic or diastolic blood pressure by $10 \mathrm{mmHg}$ [45]

2. Dyslipidemia: reduction in total cholesterol by 100 $\mathrm{mg} / \mathrm{dL}$; increase in HDL by $10 \mathrm{mg} / \mathrm{dL}$; reduction in triglycerides by 50 to $100 \mathrm{mg} / \mathrm{dL}$ [33]

3. Coronary artery disease: improvement in myocardial ischemia; reduction in coronary calcium score [34]

4. Stroke

5. Obstructive peripheral arterial disease: improvement in ankle-brachial pressure index < 0.9; arterial stenosis detected by deep-vein thrombosis ultrasound of lower limbs [35]
6. Carotid atherosclerosis: reduction in intima-media thickness or degree of carotid stenosis [33]

7. Heart failure: reduction in degree of left ventricular hypertrophy or increase in systolic function of left ventricle or improvement and/or reversal of diastolic dysfunction, detected using Doppler echocardiography [36]

8. Arrhythmia: reduction in number of events of arrhythmia, detected by 24-h Holder or stress test

9. Diabetes mellitus: reduction in glucose tolerance and insulin resistance; improvements in fasting glucose, A1C hemoglobin, oral glucose tolerance test, basal insulin, insulin curve, homeostatic model assessment $[37,38]$

10. Serum markers of cardiovascular risk: reductions in ultrasensitive C-reactive protein, homocysteine, and lipoprotein A [33];

11. Hyperthyroidism or hypothyroidism: improvement in serum levels of ultrasensitive TSH, free T4, and free T3 $[39,40]$

12. Chronic kidney disease: reduction in albuminuria, measured by glomerular filtration rate and calculated based on the Chronic Kidney Disease Epidemiology Collaboration [41]

13. Hyperparathyroidism (HPT): reduction in or normalization of serum levels of HPT [42].

\section{Discussion}

The aim of this protocol is to describe procedures related to the development of a clinical trial on the therapeutic effects of vitamin D $(50,000 \mathrm{IU}$ per week for 6 months) on depression and cardiovascular risk factors in patients with depression in care at psychiatric clinics of two universities compared to a control group receiving a placebo once a week for the same period of time.

Vitamin D supplementation in patients with depression has been shown to improve depressive symptoms, as demonstrated in three systematic reviews [21, 22, 45]. Vitamin D supplementation had a moderate, statistically significant effect in patients with clinically significant depressive symptoms or depressive disorder [22]. In another systematic review, the meta-analysis of all studies without flaws demonstrated a statistically significant improvement in depression following vitamin D supplementation, with an effect size comparable to that of antidepressant medication [18]. With the aim of evaluating the potential of nutraceuticals, including vitamin $\mathrm{D}$, as adjuvants to antidepressant therapy, an important systematic review and meta-analysis conducted in $2016 \mathrm{ob}-$ tained positive results, suggesting the modulating action of nutraceuticals with regard to several neurochemical pathways involved in depression [46].

The combination of vitamin D and traditional antidepressant treatment may lead to the optimization of 
therapy for depression with greater tolerability and without additional side effects. Moreover, the therapeutic effects of vitamin D on cardiovascular risk factors may have an impact on cardiovascular risk.

Vitamin D toxicity is a rare condition caused by an overdose of vitamin $\mathrm{D}$, in which markedly high concentrations of 25(OH)D (>150 ng/ml) are accompanied by severe hypercalcemia, hypercalciuria, and hypoparathyroidism, with very low or even undetectable parathormone (PTH) [4]. This condition is typically seen in patients receiving massive doses of vitamin D (50,000 to $1,000,000 \mathrm{IU})$ daily for several months to years $[47,48]$.

Hypervitaminosis D and hypercalcemia can have an exogenous etiology, provoked by the excessive or uncontrolled use of high oral doses of vitamin D or its analogs or metabolites. They can also be endogenous due to the excessive production of $1,25(\mathrm{OH}) 2 \mathrm{D}$ in granulomatous disorders, such as sarcoidosis, tuberculosis, lymphoma, and idiopathic infantile hypercalcemia [49]. Mutations of the 1,25(OH)2D $(\mathrm{OH}) 2$ cytochrome P450 D-24hydroxylase (CYP24A1) gene constitute another cause of vitamin D toxicity and hypercalcemia [50].

According to the Institute of Medicine (IOM) and the Endocrine Society, acute vitamin D toxicity is extremely rare in the literature, and other factors, such as calcium intake, can affect the risk of developing hypercalcemia and vitamin D toxicity. Independently of additional risk factors for vitamin D toxicity, many studies offer evidence that vitamin $\mathrm{D}$ is probably one of the least toxic liposoluble vitamins and is much less toxic than vitamin $\mathrm{A}[51,52]$.

Considering the preceding comments, the dosage of vitamin D proposed for the study (50,000 IU per week) meets the criteria established in a previous study, which reported positive results in terms of the reduction in depressive symptoms [20]. The aim of using this dosage is to minimize potential bias. The risk of toxicity is minimal, as dosages up to $10,000 \mathrm{IU}$ per day (70,000 IU per week) for 5 months did not induce signs of toxicity, such as hypercalcemia and hypercalciuria, in a previous study [29].

The results of the proposed clinical trial could contribute to the understanding of the therapeutic effects of vitamin D supplementation on depression and the risk of suicide. This study could also demonstrate the importance of investigating serum vitamin D levels in patients with depression. Regarding cardiovascular risk, the results of the study could assist in the implementation of the evaluation of cardiovascular risk factors in patients with depression in an attempt to reduce morbidity and mortality rates in this population. Another potential benefit of this clinical trial is the contribution to public policies addressing the importance of serum levels of vitamin D to the identification of patients with deficiencies in this vitamin and promoting its supplementation for the prevention of cardiovascular events, depression, and suicide.
The results and conclusions of this clinical trial will be sent to national and international symposiums as well as peer-reviewed journals.

\section{Ethics and intellectual property}

This clinical trial received approval from the Human Research Ethics Committee of the Federal University of Pernambuco (certificate number 2.464.997 on November 1, 2018).

\section{Access, re-use, and data sharing}

The datasets used and analyzed during the study will be made available by the author through any reasonable request.

\section{Storage, curation, and long-term preservation}

The datasets used and analyzed during the study will be in the custody of the researcher in charge of the project.

\section{Limitations}

The study will be conducted at only two public universities. The participants will also need to visit the outpatient clinics on a weekly basis to receive the medication, which could lead to absenteeism. To circumvent this problem, a research staff member can visit the patient's home in cases of failure to appear at the clinics.

The antidepressant medications will be determined by the psychiatrist providing care to the patient. Thus, the researchers of this clinical trial will have no control over the type or dosage of the antidepressants prescribed. This lack of standardization of antidepressant medications may be a source of bias in the research.

\section{Trial status}

For proof see http://www.ensaiosclinicos.gov.br/rg/. The trial is registered as number RBR-6yj8sj/UTN U11111217-9237 (July 23, 2018). Recruitment (screening of patients) was initiated on April 30, 2019. Thus, the study is currently in its initial phase (ongoing). The clinical trial (intervention) is expected to begin on October 1, 2019, as stipulated in the registry (RBR-6yj8sj/UTN U11111217-9237).

\section{Supplementary information}

Supplementary information accompanies this paper at https://doi.org/10 1186/s13063-019-3699-3.

Additional file 1. SPIRIT 2013 checklist: recommended items to address in a clinical trial protocol and related documents. (DOC $123 \mathrm{~kb}$ )

\section{Abbreviations}

HPT: Hyperparathyroidism; PRISMA: Preferred Reporting Items for Systematic Reviews and Meta-Analyses; SPIRIT: Standard Protocol Items:

Recommendations for Interventional Trials; SPSS: Statistical Package for the Social Sciences; UFPE: Federal University of Pernambuco; UPE: University of Pernambuco 


\section{Acknowledgements}

Not applicable.

\section{Authors' contributions}

All authors made substantial contributions to the conception of the study and participated in the preparation of the submission request. CMP conceived the study, developed the criteria, and wrote this protocol article. TPSS and EBS guided all phases of the article and performed a revision of the manuscript. All authors read and approved the final version.

\section{Authors' information}

EBS is a permanent professor of the Neuropsychiatry and Behavioral Sciences Program of the Federal University of Pernambuco. TPSS is a postdoctoral researcher of the Neuropsychiatry and Behavioral Sciences Program of the Federal University of Pernambuco. CMP is a Ph.D. student in the Neuropsychiatry and Behavioral Sciences Program at the Federal University of Pernambuco.

\section{Funding}

There are no sources of external funding. The protocol and trial are being funded by the researcher with personal resources.

\section{Availability of data and materials}

Not applicable.

\section{Ethics approval and consent to participate}

The study was carried out in accordance with the Declaration of Helsinki and received approval form the Human Research Ethics Committee of the Federal University of Pernambuco (certificate 2.464 .997 on $01 / 01 / 2018$ ). The study protocol was also retrospectively registered with the Brazilian registry of clinical trials (http://www.ensaiosclinicos.gov.br/rg/) with number RBR6yj8sj/UTN U1111-1217-9237 (July 23, 2018). Informed consent will be obtained from all study participants.

\section{Consent for publication}

All participants have agreed to the publication of the results.

\section{Competing interests}

The authors declare that they have no competing interests.

\section{Received: 11 February 2019 Accepted: 4 September 2019}

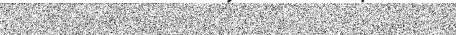

\section{References}

1. World Health Organization. Depression. Geneva: WHO; 2015.

2. Parker GB, Brotchie H, Graham RK. Vitamin D and depression. J Affect Disord. 2017:208:56-61.

3. World Health Organization. Depression: fact. Geneva: WHO; 2017. http:// www.who.int/mediacentre/factsheets/fs369/en/. Accessed 22 Jun 2018

4. Hare DL, Toukhsati SR, Johansson P, Jaarsma T. Depression and cardiovascular disease: a clinical review. Eur Heart J. 2014;351:365-72.

5. Bertone-Johnson ER. Vitamin D and the occurrence of depression: causa association or circumstantial evidence? Nutr Rev. 2009;67:481-92.

6. Cass WA, Smith MP, Peters LE. Calcitriol protects against the dopamine- and serotonin-depleting effects of neurotoxic doses of methamphetamine. Ann N Y Acad Sci. 2006;1074:261-71.

7. Ju S-Y, Lee Y-J, Jeong SN. Serum 25-hydroxyvitamin D levels and the risk of depression: a systematic review and meta-analysis. J Nutr Health Aging. 2013;17:447-55

8. Adamson J, Lally J, Gaughran F, Krivoy A, Allen L, Stubbs B. Correlates of vitamin $D$ in psychotic disorders: a comprehensive systematic review. Psychiatry Res. 2017;249:78-85.

9. Fond G, Faugere M, Faget-Agius C, Cermolacce M, Richieri R, Bover L, et al. Hypovitaminosis $D$ is associated with negative symptoms, suicide risk, agoraphobia, impaired functional remission, and antidepressant consumption in schizophrenia. Eur Arch Psychiatry Clin Neurosci. 2018. https://doi.org/10.1007/s00406-018-0932-0.

10. Kocovska E, Gaughran F, Krivoy A, Meier UC. Vitamin-D deficiency as a potential environmental risk factor in multiple sclerosis, schizophrenia, and autism. Front Psychiatry. 2017;8:1-11.
11. Casseb GAS, Kaster MP, Rodrigues ALS. Potential role of vitamin D for the management of depression and anxiety. CNS Drugs. 2019;33:619-37.

12. Schneider B, Weber B, Frensch A, Stein J, Fritz J. Vitamin D in schizophrenia, major depression and alcoholism. J Neural Transm. 2000;107(7):839-42.

13. Fond G, Godin O, Schürhoff F, Berna F, Bulzacka E, Andrianarisoa M, et al. Hypovitaminosis $D$ is associated with depression and anxiety in schizophrenia: results from the national FACE-SZ cohort. Psychiatry Res. 2018;270:104-10.

14. Kjærgaard M, Joakimsen R, Jorde R. Lowserum 25-hydroxyvitamin D levels are associated with depression in an adult Norwegian population. Psychiatry Res. 2011;190:221-5.

15. Stokes CS, Grünhage F, Baus C, Volmer DA, Wagenpfeil S, Riemenschneider $\mathrm{M}$, et al. Vitamin D supplementation reduces depressive symptoms in patients with chronic liver disease. Clin Nutr. 2016;35:950-7.

16. Bahrami A, Mazloum SR, Maghsoudi S, Soleimani D, Khayyatzadeh SS, Arekhi $\mathrm{S}$, et al. High dose vitamin D supplementation is associated with a reduction in depression score among adolescent girls: a nine-week followup study. J Diet Suppl. 2018;15:173-82.

17. Jhee JH, Kim H, Park S, Yun HR, Jung SY, Kee YK, et al. Vitamin D deficiency is significantly associated with depression in patients with chronic kidney disease. PLOS One. 2017:12:e0171009.

18. Hoogendijk WJ, Lips P, Dik MG, Deeg DJ, Beekman AT, Penninx BW. Depression is associated with decreased 25 -hydroxyvitamin $D$ and increased parathyroid hormone levels in older adults. Arch Gen Psychiatry. 2008;65:508-12.

19. Zhu D, Zhao M, Zhang W, Zhang B, Yang Y, Zhang Y, et al. The relationship between serum concentration of vitamin $\mathrm{D}$, total intracranial volume, and severity of depressive symptoms in patients with major depressive disorder. Front Psychiatry. 2019;10(322):1-7.

20. Sepehrmanesh Z, Kolahdooz F, Abedi F, Mazroii N, Assarian A, Asemi Z, et al. Vitamin D supplementation affects the beck depression inventory, insulin resistance, and biomarkers of oxidative stress in patients with major depressive disorder: a randomized, controlled clinical trial. J Nutr. 2016;146:243-8.

21. Spedding S. Vitamin D and depression: a systematic review and metaanalysis comparing studies with and without biological flaws. Nutrients. 2014;6:1501-18

22. Shaffer JA, Edmondson D, Wasson LT, Falzon L, Homma K, Ezeokoli N, et al. Vitamin D supplementation for depressive symptoms: a systematic review and meta-analysis of randomized controlled trials. Psychosom Med. 2014;76: 190-6.

23. Montgomery SA, Asberg M. Anew depression scale designed to be sensitive to change. Br J Psychiatry. 1979;134:382-9.

24. Organização Mundial da Saúde. CID-10: Classificação Estatística Internacional de Doenças com disquete, vol. 1. São Paulo: EDUSP; 1994.

25. Dumont CS, Gillette-Guyonnet S, Andrieu C, Cantet PJ, Ousset B, Vellas B. Rapide du Mini Mental State Examination: étude REAL. FR, La Revue de Médecine Interne. 2003;24:345s-50s.

26. Posner K, Brown GK, Stanley B, Brent DA, Yershova KV, Oquendo MA, et al. The Columbia-Suicide Severity Rating Scale: initial validity and internal consistency findings from three multisite studies with adolescents and adults. Am J Psychiatry. 2011;168:1266-77.

27. Bandeira F, Griz L, Freese E, Lima DC Thé AC, Diniz ET, et al. Vitamin D deficiency and its relationship with bone mineral density among postmenopausal women living in the tropics. Ara Bras Endocrinol Metab. 2010;54:227-32.

28. Pathak S, Nisar MA, Rosenthal DS. Vitamin D deficiency (VDD) and major depressive disorder (MDD): a causal or casual association? J Endrocr Soc 2012; http://press.endocrine.org/doi/abs/10.1210/endo-meetings.2012.BCH.3. SAT-377. Accessed 18 Oct 2016

29. Heaney RP, Davies KM, Chen TC, Holick MF, Barger-Lux MJ. Human serum 25-hydroxycholecalciferol response to extended oral dosing with cholecalciferol. Am J Clin Nutr. 2003;77:204-10.

30. Sepehrmanesh Z, Kolahdooz F, Abedi F, Mazroii N, Assarian A, Asemi Z. Vitamin D supplementation affects the Beck Depression Inventory, insulin resistance, and biomarkers of oxidative stress in patients with major depressive disorder: a randomized, controlled clinical trial. J Nutr. 2016;146:243-8.

31. Brazilian Association of Companies in Research. Criterion of economic classification in Brazil. São Paulo: Brazilian Association of Companies in Research; 2015. 
32. Whelton PK, Carey RM, Aronow WS, Casey DE Jr, Collins KJ, Dennison Himmelfarb C. ACC/AHA/AAPA/ABC/ACPM/AGS/APhA/ASH/ASPC/NMA/ PCNA guideline for the prevention, detection, evaluation, and management of high blood pressure in adults. J Am Coll Cardiol. 2017. https://doi.org/10. 1016/j.jacc.2017.11.006.

33. Faludi AA, Izar MCO, Saraiva JFK, Chacra APM, Bianco HT, Afiune Neto A et al. Atualização da Diretriz Brasileira de Dislipidemias e Prevenção da Aterosclerose - 2017. Arq Bras Cardiol. 2017;109(2Supl.1):1-76.

34. Cesar LA, Ferreira JF, Armaganijan D, Gowdak LH, Mansur AP, Bodanese LC, et al. Diretriz de Doença Coronária Estável. Arq Bras Cardiol. 2014; 103(2Supl.2): 1-59.

35. Presti C, Miranda F Jr, Casella IB, De Luccia N, Covre MR. Projeto diretrizes SBACV: Doença arterial periferia obstrutiva de membros inferiores: diagnóstico e tratamento. Recife: Sociedade Brasileira de Angiologia e Cirurgia Vascular; 2015

36. American Heart Association. Ejection fraction heart failure measurement. https://www.heart.org/en/health-topics/heart-failure/diagnosing-heartfailure/ejection-fraction-heart-failure-measurement. Accessed 25 May 2019.

37. William T, Cefalu MD. Standards of medical care in diabetes-2017: American Diabetes Association (ADA). Diabetes Care 2017;40 (Supp 1):S1-S134.

38. Milech A, Perez Angelucci A, Golbert A, Matheus A, Carrilho Faria AJ, Ramalho AC, et al. Diretrizes da Sociedade Brasileira de Diabetes (2015-2016). São Paulo: A.C. Farmacêutica; 2016.

39. Maia AL, Scheffel RS, Meyer EL, Mazeto GM, Carvalho GA, Graf H, et al. The Brazilian consensus for the diagnosis and treatment of hyperthyroidism: recommendations by the Thyroid Department of the Brazilian Society of Endocrinology and Metabolism. Arq Bras Endocrinol Metabol. 2013;57:205-24.

40. Brenta G, Vaisman M, Sgarbi JA, Bergoglio LM, Andrada NC, Bravo PP, et al. Clinical practice guidelines for the management of hypothyroidism. Arq Bras Endocrinol Metab. 2013;57:265-91.

41. KDIGO. Clinical practice guideline for the evaluation and management of chronic kidney disease. Kidney Int Suppl. 2013;3:1-150.

42. Bandeira F, Griz L, Chaves N, Carvalho NC, Borges LM, Lazaretti-Castro M, et al. Diagnosis and management of primary hyperparathyroidism: a scientific statement from the Department of Bone Metabolism, the Brazilian Society for Endocrinology and Metabolism. Arq Bras Endocrinol Metab. 2013;57:406-24.

43. Arnett DK, Blumenthal RS, Albert MA, Buroker AB, Goldberger ZD, Hahn EJ, et al. 2019 ACC/AHA guideline on the primary prevention of cardiovascular disease: a report of the American College of Cardiology/American Heart Association Task Force on clinical practice guidelines. J Am Coll Cardiol. 2019:26029. https://doi.org/10.1016/j.jacc.2019.03.010.

44. Azevedo TA, Moreira MLV, Nucera ACSN. Cardiovascular risk estimation by the ASCVD Risk Estimator application in a university hospital. Int J Cardiovasc Sci. 2018;31:492-8

45. Malachias Bolívar MV, Souza Weimar KSB, Plavnik FL, Rodrigues CIS, Brandão AA, Neves MFT. 7a Diretriz Brasileira de Hipertensão Arterial. Braz J Hipertens. 2017:24:2-91.

46. Sarris J, Murphy J, Mischoulon D, Papakostas Gl, Fava M, Berk M, Ng CH. Adjunctive nutraceuticals for depression: a systematic review and metaanalyses. Am J Psychiatry. 2016;173:575-87.

47. Holick MF. Vitamin D is not as toxic as was once thought: a historical and an up-to-date perspective. Mayo Clin Proc. 2015;90:561-4.

48. Holick MF. The vitamin D deficiency pandemic: approaches for diagnosis, treatment and prevention. Metab Syndr Relat Disord. 2017;18:153-65.

49. Marcinowska-Suchowierska E, Kupisz-Urbańska M, Łukaszkiewicz J, Płudowski P, Jones G. Vitamin D toxicity—a clinical perspective. Front Endocrinol (Lausanne). 2018;9:550

50. Tebben PJ, Singh RJ, Kumar R. Vitamin D-mediated hypercalcemia: mechanisms, diagnosis, and treatment. Endocr Rev. 2016;37:521-47.

51. OIM (Instituto de Medicina). Dietary reference intakes for calcium and vitamin D. Washington, DC: National Academies Press; 2011. p. 1-1115.

52. McCullough PJ, Lehrera DS, Amend J. Daily oral dosing of vitamin $D_{3}$ using 5000 to 50,000 international units a day in long-term hospitalized patients: insights from a seven year experience. J Steroid Biochem Mol Biol. 2019:189:228-39.

\section{Publisher's Note}

Springer Nature remains neutral with regard to jurisdictional claims in published maps and institutional affiliations.

Ready to submit your research? Choose BMC and benefit from:

- fast, convenient online submission

- thorough peer review by experienced researchers in your field

- rapid publication on acceptance

- support for research data, including large and complex data types

- gold Open Access which fosters wider collaboration and increased citations

- maximum visibility for your research: over $100 \mathrm{M}$ website views per year

At $\mathrm{BMC}$, research is always in progress.

Learn more biomedcentral.com/submissions 